\title{
OBJECTIVE ASSESSMENT FOR CHARACTERISING THE FLATNESS OF GARMENT SEWING STITCHES
}

\author{
Jiang Hongxia ${ }^{1}$, Liu Jihong ${ }^{1}$, Chai Zhilei ${ }^{1,2}$, Wang Chunxia ${ }^{3}$, Zhang Mingxia ${ }^{1}$ \\ ${ }^{1}$ Key Laboratory of Eco-textiles, Ministry of Education, Jiangnan University, Wuxi, Jiangsu, China \\ ${ }^{2}$ School of Internet of Things, Jiangnan University, Wuxi, Jiangsu, China \\ ${ }^{3}$ Yancheng Institute of Technology, Yancheng, Jiangsu, China \\ E-mail: jianghongxiatex@hotmail.com, liujihongtex@hotmail.com, zlchai@jiangnan.edu.cn, cxwang@mail.dhu.edu.cn
}

\begin{abstract}
:
In this paper, a novel classification method of assessing garment sewing stitch based on amended bi-dimensional empirical mode decomposition (ABEMD) has been introduced. Two parameters that characterise garment sewing stitch, average area and standard deviation, have been defined based on the grey value of pixels. Experimental results showed that when the window size is $512 \times 128$ pixels with regard to average area, the threshold can be decided as 6.00, 5.50, 5.30 and 4.00 for five different grades, respectively. Meanwhile, with regard to standard deviation, the threshold can be decided as 48.00, 40.00,30.00 and 20.00, respectively. It is demonstrated that the parameters are effective in discriminating sewing stitch images in terms of the grades when used as inputs for the $A B E M D$. The performance of the algorithm on different garment status is significantly reliable.
\end{abstract}

\section{Keywords:}

Assessment, Sewing stitch, Flatness, Characterisation

\section{Introduction}

The garment appearance is affected by a number of factors determined by the technology of the garment manufacturing process. The garment sewing stitches is one of the factors that impact garments quality, aesthetic aspect and so on. Conventionally, the garment sewing stitches is a vital performance characteristic of a garment. Researchers have begun to study the performance of garment sewing stitches. Amirbayat and Morton [1,2] used the energy method to find the stable state of sewing stitches and occurrence reasons of seam puckering. Zavec et al. [3] presented the impact of fabric mechanical properties on the quality of sewing stitch appearance and work-piece flotation by machine learning methods. The theoretical analysis of the sewing stitch slippage mechanism has been performed by the literature analysis and the results of experimental studies which were obtained $[4,5]$. A linear three-dimensional finite-element analysis in ABAQUS is used to model sewing stitch formation under sewing thread tension [6]. Stylios et al. tried to predict quality of sewing stitches using thickness, weight, and weft and warp bending stiffness as inputs to a back propagation neural network technique [7]. Fan and Leeuwner [8] have worked on the parameters involved in the physical and mechanical properties of the sewing thread that affect sewing stitch. Dobilaite and Juciene [9] reported that the mechanical properties of sewing thread were obtained by performing tensile testing research. The effect of exerted forces by sewing thread on fabric in the seam and stitch element is investigated by simulation method [10-12]. Samuel and Poojitha [13] provided a simple system for online measuring the actual thread consumed for every 10 stitches. Rengasamy [14] studied dynamic sewing tension by a tension probe on sewing threads with different structures.
Meanwhile, the flatness of garment sewing stitches in sewing and finishing procedures is also one of important properties. In general, the flatness of garment sewing stitches is evaluated by visual examination of sewing stitch samples performed by trained experts in accordance with the criterion based on the AATCC 88B-2011 standard method [15]. The evaluation is done by three observers who compare three test specimens at a time with photographs under an overhead lighting [16]. Since subjective evaluation of the sewing stitches depends on human vision, perception, knowledge and experience of judges, sometimes even for specialists it is difficult to decide on the grade of sewing stitches. Therefore, researchers have concentrated on developing an objective assessment and grading method for sewing stitches in order to replace the subjective assessment method. Many instrumental methods have been developed and introduced to the textile industry to meet the need for more reliable measurement of sewing stitches.

Kim and Kim [17] proposed an objective evaluation of the seam pucker for the worsted and wool/polyester blend fabrics according to mechanical property by measuring results using KES-FB and FAST systems. It was shown that low presser foot pressure and shuttle tension make good sewing stitches, and inverse high sewing speed and shuttle tension make bad sewing stitches. The curve features provide a practice method for grading sewing stitches by measuring these parameters based on the characteristics. Dobilaite and Juciene [18] provided an inverse evaluation method by analysing the distribution dynamics of the waves made by the place of the stitching of different technological modes. Fan and Liu [19] reported on the development of an objective method for evaluating the degree of garment sewing stitch by a three-dimensional laser scanning 
system. From sewing stitch profile, a physical parameter, $\log \left(\sigma^{2}\right)$, is linearly related to the subjective pucker grade.

Sewing stitch is an area which is now receiving particular attention especially with the development of vision system which is prone to spotting deformations during garment manufacture. Styliosa and Sotomia $[20,21]$ reported on the development of a new measuring procedure for sewing stitch which is based on computer vision. The procedure is objective and is focused on the aesthetic property of sewing stitch assembly. The results are presented in two parts; Part I reported on the development of a cognitive model for the measurement of sewing stitch and Part II explains the model's implementation by using the computer 'vision' system. Amirbayata [22] presented the results of experiments on seams sewn under constant sewing conditions when the mechanical properties of the plies are different. By using plies of the same fabric along different directions, the effects of the mean properties of the plies as well as their incompatibility are studied, while the other two important factors, the fabric thickness and the compressive modulus, are kept unchanged. The extensibility and strength of such seams will be studied in the second part of this series.

The curve geometrical characteristics of garment sewing stitch, such as curve area and amplitude, are the important features that change a sewing stitch apparent. Mousazadegan et al. [23] introduced a novel approach for seam pucker analysis based on wave shape parameters. Results analysis showed that there is a high linear relation with high correlation between all pucker indexes and subjective pucker evaluation. In this study, we use image processing to analyse the sewing stitch in detail and propose the future of sewing stitch for grading the samples. In fact, imaging technology $[24,25]$ has been used to generate surfaces or profiles from which fabric appearance can be detected. Various digital image analysis methods have been adopted for automatic wrinkle characterisation and grading based on two-dimensional texture information and other applications [26-31].

Of these images processing methods, empirical mode decomposition (EMD), developed originally by Huang et al. [32], is a totally data-driven empirical method related to the locations of extra points and zero-crossings. As a twodimensional expansion of EMD, bi-dimensional empirical mode decomposition (BEMD) can be used to decompose characteristic parameters in frequency domain for curves as a non-stationary and nonlinear algorithm. The BEMD has been applied widely in many fields, such as noise reduction [33], image segmentation [34], texture synthesis [35], edge detection [36] and so on. BEMD can break up an image as a two-dimensional signal into a set of bi-dimensional intrinsic mode functions (BIMF) and a set of residue and allows each domain to be studied for surface. Therefore, BEMD is a powerful tool to remove unwanted information associated with weave structures and illumination variations, which are separable at different decomposing levels or frequency domains [37].

Using this approach, we will explore a reliable way for sewing stitch evaluation by BEMD. It is supposed that images of garment sewing stitch are composed of different kinds of components including fibre texture, fabric texture, sewing stitch texture and so on, and the image can be segmented to different frequency domains by BEMD. Therefore, it is possible to analyse the sewing stitch in more detail and extract the parameters to establish a firm base for the analysis of different grades. It is also possible to develop an objective method based on the objective information of BEMD. In this study, first, BEMD and the corresponding algorithm are introduced. Two parameters that characterise sewing stitches are then defined based on the grey value of pixels. After this, the objective grading method is deduced.

\section{Method}

\section{Image Acquisition}

A Microtek flat scanner was used to capture reflection images of garment sewing stitches in RGB mode. The resolution is set at $100 \mathrm{dpi}$. To reduce colour differentiation on the garment's appearance, the capture region was chosen far from the edge of the fabric, and the surface of garment sewing stitch should be clear. To validate the effect of the algorithm of garment sewing stitch evaluation system, five fabrics with deferent degrees of seam smoothness were chosen to test the system. Each sample was graded with AATCC 88B-2011, and then imaged by the system. Five sample images were selected at each grade.

\section{Image Process}

The fabric images of treated samples were converted to grey mode. The greyscale images were then normalised by dividing the pixels with the maximum value. After this, amended bidimensional empirical mode decomposition (ABEMD) is introduced to decouple the surface of sewing stitches from the image of garment sewing stitch. The intrinsic mode function (IMF) was generated using BEMD according to spatial instantaneous frequency for image decomposition.

\section{Sifting Process for Sewing Stitches by ABEMD}

Huang et al. [32] provided an algorithm of EMD as a powerful tool for adaptive multi scale analysis of non-stationary signals. Damerval et al. [38] have written a similar algorithm for BEMD using for EMD. Median filter was applied before the BEMD started because there is noise in the image. Therefore, we call the algorithm as ABEMD. The decomposition process of bi-dimensional IMF (BIMF) is called sifting process (SP). The image can be expressed as bi-dimensional nonlinear signal $f(\mathrm{i}$, j) $(0 \leq f(\mathrm{i}, \mathrm{j}) \leq 255)$ and $f(\mathrm{i}, \mathrm{j})$ are integers. In the $f(\mathrm{i}, \mathrm{j}), \mathrm{i}(1 \leq \mathrm{i} \leq \mathrm{M})$ expresses the number of row and $j(1 \leq j \leq N)$ expresses number of column. The algorithm for sifting on a greyscale image is implemented as follows.

Step 1: Smooth the $f(i, j)$ in spatial filter.

Step 2: Set residue matrix $r(i, j)=f(i, j)$ and number for BIMF.

Step 3: Get subset $I_{\text {max }}(m, n)$ and $I_{\text {min }}(m, n)$ by finding the 
positions and amplitudes of all local maxima and minima of the signal $r(\mathrm{i}, \mathrm{j})$.

Step 4: Create the upper and lower enveloping surface by interpolating the subset $I_{\max }(m, n)$ and $I_{\min }(m, n)$, respectively. The data of $m$ and $n$ are decided by the number of all local maxima and minima. Denote the upper and lower enveloping surface by $e_{\text {upper }}(i, j)$ and $e_{\text {lower }}(i, j)$, respectively.

Step 5: Calculate the mean surface between the upper and lower envelope by Eq.1. Denote the mean surface by $e_{\text {mean }}(i, j)$.

$$
e_{\text {mean }}(i, j)=\frac{e_{\text {upper }}(i, j)+e_{\text {lower }}(i, j)}{2}
$$

Step 6: Subtract the mean surface from the input residue matrix $r(\mathrm{i}, \mathrm{j})$ and get oscillating signal $c / k(\mathrm{i}, \mathrm{j})=r(\mathrm{i}, \mathrm{j})-e_{\text {mean }}(\mathrm{i}, \mathrm{j})$.

Step 7: When $e_{\text {mean }}(\mathrm{i}, \mathrm{j})$ meets the stopping criterion, $c /(\mathrm{i}, \mathrm{j})=c / k(\mathrm{i}, \mathrm{j})$ becomes IMF I, and $r(\mathrm{i}, \mathrm{j})-c / k(\mathrm{i}, \mathrm{j})$ becomes the residue I. Otherwise, set $r(\mathrm{i}, \mathrm{j})=c l k(\mathrm{i}, \mathrm{j})$ and repeat the processes, beginning at Step 2.

Step 8: The next IMF is found by starting over from Step 2, now setting the residue $r(\mathrm{i}, \mathrm{j})-c /(\mathrm{i}, \mathrm{j})$ as the input matrix $r(\mathrm{i}, \mathrm{j})$ until the recycle number meets number for BIMFs.

\section{Sewing Stitch Assessment}

\section{Decomposing of IMF and Residue}

To explain the image process of ABEMD, sample I was selected as shown in Figure 1(a). There is a typical sewing stitch in the image. Before extracting BIMF during sifting process, we used bi-dimensional order-statistic filtering to decrease the number of maximum pixels or minimum pixels. Bi-dimensional orderstatistic filtering of sample I in two dimensions was performed using the default 3-by-3 neighbourhood and the result is shown in Figure 1(b). In the bi-dimensional order-statistic filtering, each pixel in $r(i, j)$ was replaced by the orderth pixel in the sorted set of neighbours using Eq.2.

$$
f(i, j)=\underset{(s, t) \in s_{x y}}{\operatorname{order}}\{\hat{f}(s, t)\}
$$

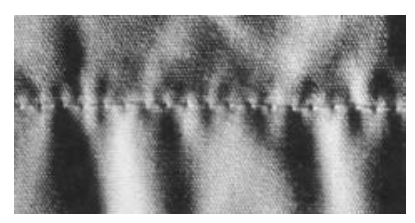

(a)

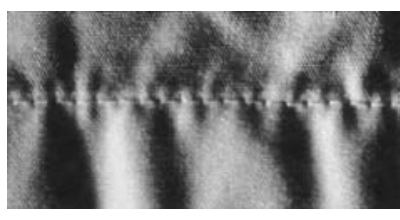

(b)
Figure 1. Image of sample I (a) Source image, (b) Result of bidimensional order-statistic filtering.

For analysing $I_{\max }(m, n)$, each output pixel in Figure 2 contains the maximum value in the 3-by-3 neighbourhood around the corresponding pixel in Figure 1(2). For analysing $I_{\text {min }}(m, n)$, each output pixel contains the minimum value in the 3 -by-3 neighbourhood around the corresponding pixel. The algorithm provided noise reduction capabilities with considerably less blurring.

After that we set out 10 line equal to the inner 11th line. That means the first top 10 line is equal to the 11 th line and the first bottom 10 line is equal to the bottom 11th line. At same time, the first left 10 line is equal to the left 11 th line and the right 10 line is equal to the 11th line from right size. The result is shown in Figure 2. As result, there are four equal points at each corner of four corners.

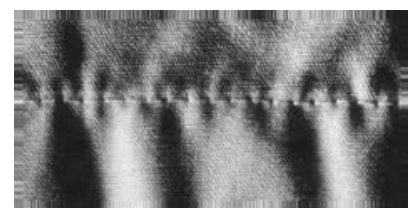

Figure 2. Set outline of source image.

We can get subset $I_{\text {max }}(m, n)$ and $I_{\text {min }}(m, n)$ by finding the positions and amplitudes of all local maxima and minima of the signal $r(\mathrm{i}, \mathrm{j}) . /(\mathrm{i}, \mathrm{j})$ is a maximum pixel if the $/(\mathrm{i}, \mathrm{j})$ meets one of the two conditions. One condition is that $l(\mathrm{i}, \mathrm{j})$ is greater than or equal to the value of $l(\mathrm{i}, \mathrm{j})$ at the eight nearest neighbours of $I(\mathrm{i}, \mathrm{j})$. The other condition is that $I(\mathrm{i}, \mathrm{j})$ is greater than one of the value of $l^{\prime}(\mathrm{i}, \mathrm{j})$ at the eight nearest neighbours of $l(\mathrm{i}, \mathrm{j})$ at least. The upper enveloping surface $e_{\text {upper }}(i, j)$ was created by interpolating the subset $I_{\max }(\mathrm{m}, \mathrm{n})$.

Meanwhile, $I(\mathrm{i}, \mathrm{j})$ is a minimum pixel if the $I(\mathrm{i}, \mathrm{j})$ meets one of the two conditions. One condition is that $I(\mathrm{i}, \mathrm{j})$ is less than or equal to the value of $l^{\prime}(\mathrm{i}, \mathrm{j})$ at the eight nearest neighbours of $I(\mathrm{i}, \mathrm{j})$. The other condition is that $I(\mathrm{i}, \mathrm{j})$ is less than one of the value of $l^{\prime}(\mathrm{i}, \mathrm{j})$ at the eight nearest neighbours of $I(\mathrm{i}, \mathrm{j})$ at least. As shown in Figure 3, the lower enveloping surface $e_{\text {lower }}(\mathrm{i}, \mathrm{j})$ was created by interpolating the subset $I_{\text {min }}(m, n)$. Figure $3(a)$ shows the three-dimensional figure of part of $r(i, j)(30<i<100,30<j<100)$. Figure $3(b)$ shows $e_{\text {upper }}(i, j)$ and $e_{\text {lower }}(\mathrm{i}, \mathrm{j})$. From the Figure 3 , we find $e_{\text {upper }}(\mathrm{i}, \mathrm{j})$ and $e_{\text {lower }}(\mathrm{i}, \mathrm{j})$ are created correctly. The $e_{\text {mean }}(\mathrm{i}, \mathrm{j})$ shown in Figure 4 was calculated by Eq.1.

$c l k(i, j)$ was acquired recently by subtracting the mean surface from the input residue matrix $r(\mathrm{i}, \mathrm{j})$ until $e_{\text {mean }}(\mathrm{i}, \mathrm{j})$ meets the stopping criterion. Finally, BIMFs and residues were created. Figure 5 shows the result of three-dimensional IMF1 and Figure 6 shows the result of three-dimensional residues 1 .

\section{Properties of BIMF and Residues in ABEMD}

In the algorithm of $A B E M D$, the BIMFs consist of oscillation modes in a higher spatial frequency interval. Meanwhile, the residues consist of oscillation modes in a lower spatial frequency interval. The surface of sewing stitches normally appears in lower frequency domains than the noise signal and fabric texture (weave patterns, yarns, etc.), and in higher frequency domains than the oblique lighting. Considering a garment sewing stitches image as a superposition of assorted components, we implement ABEMD to breakdown the components into different BIMFs and residues. 
(a)

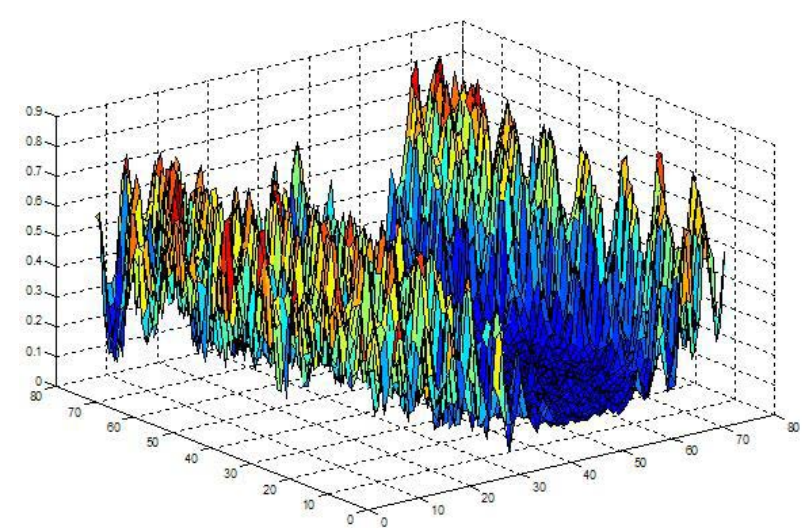

(b)

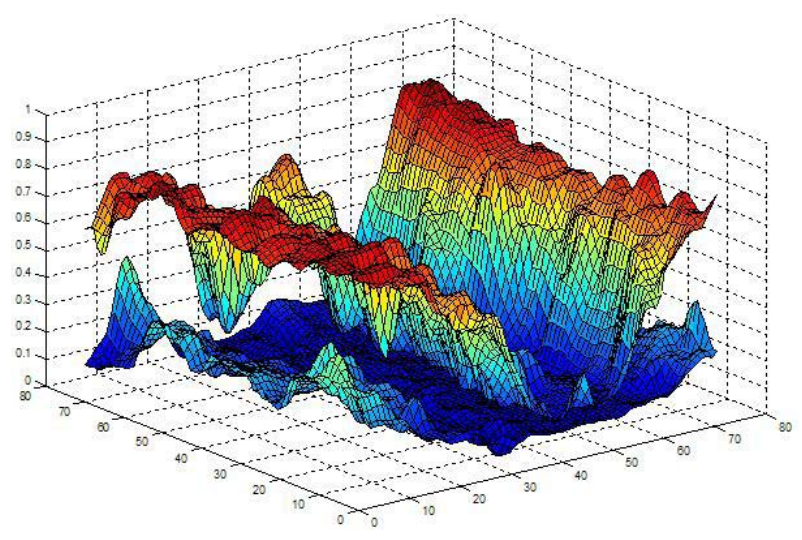

Figure 3. Three-dimensional results (a) $r(\mathrm{i}, \mathrm{j}),(\mathrm{b}) e_{\text {upper }}(\mathrm{i}, \mathrm{j})$ and $e_{\text {lower }}(\mathrm{i}, \mathrm{j})$

In the analysis, the image is decomposed into two BIMFs and residues. After the trial tests with the original images of sewing stitches, it was found that only the BIMF and residue at decomposing level 1 were needed for sewing stitch characterisation. Components in other domains were either insignificant or irrelevant in representing signals. We found that $A B E M D$ is totally data driven, self-adaptable and capable of grading sewing stitches. The property is distinguished from traditional spectral transform methods.

\section{Expression of Sewing Stitch Feature}

There are many kinds of useful wrinkling information. In this study, two types of sewing stitch parameters were defined from different perspectives: The average area of a curved surface (AACS) and the standard deviation of a curved surface (SDCS).

AACS: A quantitative measure to reflect the texture content information. The AACS is given by:

$$
A_{m n}=\frac{1}{m n} \int_{D} \int_{D} \sqrt{1+\left(r_{x}^{\prime}\right)^{2}+\left(r_{y}^{\prime}\right)^{2}} d x d y
$$

where $m$ and $n$ are the width and height of the source image, $r_{\mathrm{x}}^{\prime}$ is derivative of $r(\mathrm{i}, \mathrm{j})$ in $x$ direction, $r_{\mathrm{y}}^{\prime}$ is derivative of $r(\mathrm{i}, \mathrm{j})$ in $y$ direction. The $A_{m n}$ expresses the average area for one pixel. The greater the area $A_{m n}$, the lower the degree of sewing stitch.

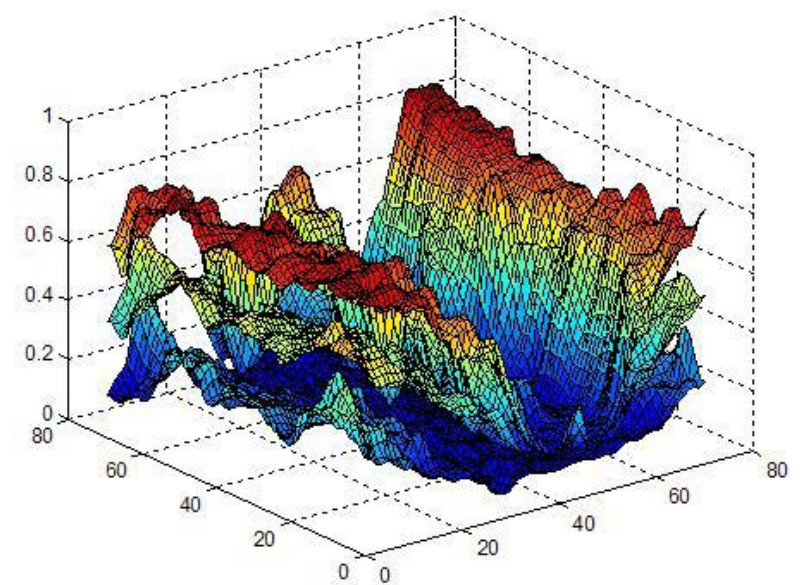

Figure 4. $e_{\text {mean }}(i, j)$ calculated from Figure $3(b)$

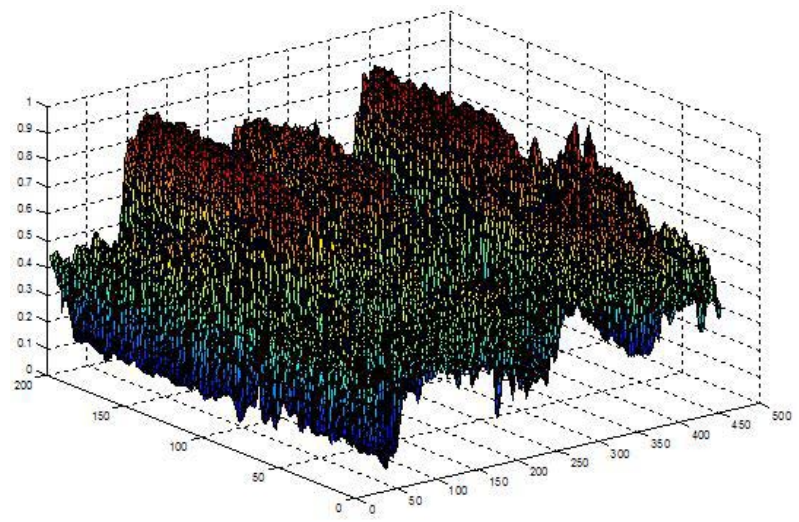

Figure 5. The result of three-dimensional IMF1

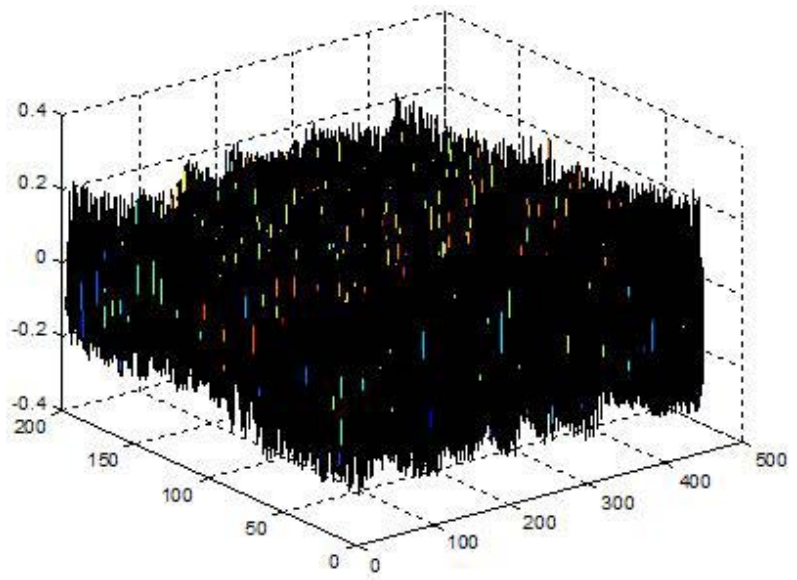

Figure 6. The result of three-dimensional residues 1

SDCS: The SDCS is given by,

$$
s=\sqrt{\frac{1}{M N-1} \sum_{i=1}^{M} \sum_{j=1}^{N}\left(r_{i, j}-\overline{r_{i, j}}\right)^{2}}
$$

where $\overline{r_{i, j}}=\frac{1}{M N} \sum_{i=1}^{M} \sum_{j=1}^{N} r_{i, j}$

and $M$ and $N$ are the width and height of the image. The $s$ expresses degree of coarseness. The greater the standard deviation $s$, the higher is the degree of coarseness. 


\section{Experimental Results}

Validation measurements were accomplished to verify whether the proposed ABEMD can appropriately detect the degree of sewing stitches and whether extracted characteristics can effectively distinguish the difference in the levels of sewing stitches. We selected five $25.4 \times 50.8 \mathrm{~mm}^{2}\left(1 \times 2 \mathrm{in}^{2}\right)$ white cotton fabrics with the same plain weave structure and only one line sewing stitch on the surface. The five samples were sewn in different levels of wrinkle of sewing stitches. The samples were graded by a special evaluator and then immediately imaged using the flat scanner. After this, sewing stitch detection was accomplished and information of sewing stitches was acquired.

Figure 7 displays the surfaces of the five samples. A sample 1 as shown (a) has a wrinkle-heavy sewing stitch. Sample 2 as shown (b) has smooth and wrinkle part of sewing stitch. Sample 3 as shown (c) is a sewing stitch with moderate wrinkles while sample 4 as shown (d) is a relatively flat sewing stitch, with only some light wrinkles, and sample 5 as shown (e) contains mostly smooth ones.

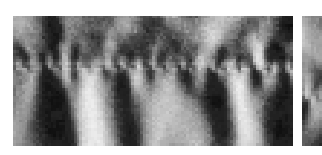

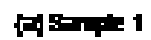

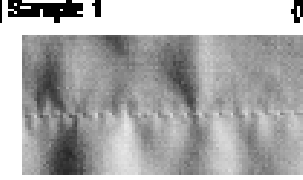

ats 5

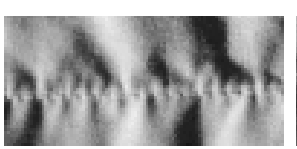

aj: 5

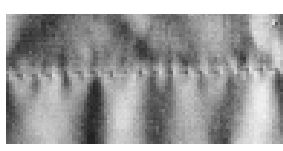

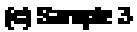

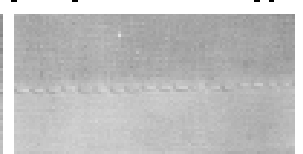

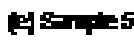

Figure 7. Five samples with different wrinkle degree of sewing stitch

Figure 8 (a) and (b) show two change trends of sewing stitch parameters. Figure 8 (a) illustrates the distributions of AACS of a curved surface calculated by the expression of Eq.3 provided. Figure 8 (b) illustrates the distributions of the SDCS of a curved surface calculated by the expression of Eq.4 and Eq.5 provided.

(a)

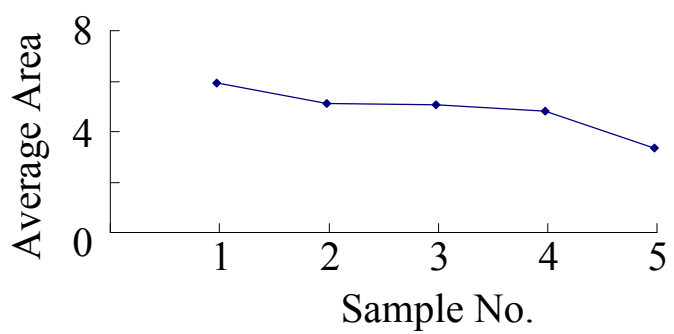

(b)

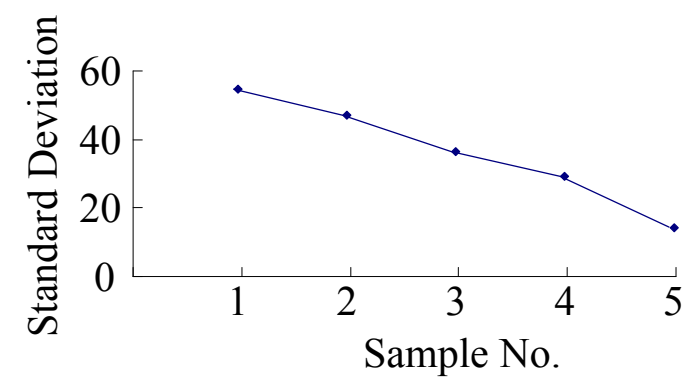

Figure 8. (a) Distribution of AACS (b) Distribution of SDCS
Both of the figures illustrate that the plots of wrinkle features extracted from the five sample surfaces have correct relationship with degree of sewing stitch. The wrinkle-heavy sample (sample 1) has greater average area and standard deviation than those of the relatively flat sewing stitch samples (samples 2, 3, 4 and 5). This is because a wrinkle-heavy surface often contains many fluctuant curve surfaces that finally contribute to increase the area and standard deviation. Figures 7 and 8 also show that samples 2, 3 and 4 have similar area although there is a trend of becoming smaller. However, there are distinguished differences in standard deviation among samples 2, 3 and 4. The greater the area or standard deviation a sample has, the lower degree a sample and, conversely, the smoother the wrinkles, the less the area or standard deviation. Two parameters have a significant correlation with the visual rating.

\section{Discussion}

The status and position affect the calculated result; therefore, we must discussion these factors.

\section{The Position of Sewing Stitch}

As shown in Figure 9, there are three typical positions of sewing stitch in the sample of garment sewing stitch image. The reference position is diagrammed as shown in Figure 9(a). There are two properties of sewing stitch in Figure 9(a). The first one is that the sewing stitch is in about the middle position of sample of fabric. The other one is parallel to horizontal diagram or the page bottom. The above and under positions are shown in Figure 9(b) and (c), respectively. The obvious characteristic is that the sewing stitch is above or under the reference position of the fabric image. Table 1 lists the results of Eigen values of samples with sewing stitch in different positions. The window size is $512 \times 128$ pixels.

(a)

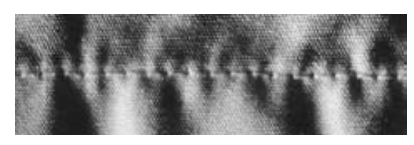

(b)

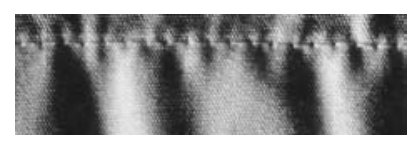

(c)

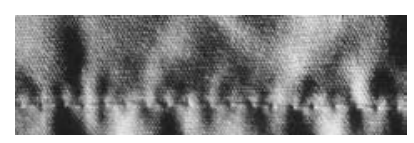

Figure 9. Three typical positions of sewing stitch

From Table 1, we can find that the Eigen values change along with position. This is caused fluctuant change around sewing stitch by cutting out fabric image. However, for average area or standard deviation there are obvious contrasts among five 
Table 1 Eigen values for three typical positions of sewing stitch

\begin{tabular}{|c|c|c|c|c|c|c|c|c|c|c|}
\hline \multirow{2}{*}{ Position } & \multicolumn{4}{|c|}{ Average area } & \multicolumn{5}{|c|}{ Standard deviation } \\
\cline { 2 - 11 } & $\mathbf{1}$ & $\mathbf{2}$ & $\mathbf{3}$ & $\mathbf{4}$ & $\mathbf{5}$ & $\mathbf{1}$ & $\mathbf{2}$ & $\mathbf{3}$ & $\mathbf{4}$ & $\mathbf{5}$ \\
\hline Above & 6.83 & 5.85 & 5.45 & 5.22 & 3.52 & 49.05 & 45.03 & 35.80 & 26.65 & 12.85 \\
\hline Reference & 6.88 & 5.84 & 5.40 & 5.15 & 3.26 & 51.95 & 46.09 & 37.53 & 28.42 & 13.73 \\
\hline Under & 6.74 & 5.65 & 5.32 & 4.98 & 3.05 & 52.81 & 46.11 & 37.63 & 29.34 & 12.13 \\
\hline
\end{tabular}

Table 2 Results under different window size

\begin{tabular}{|c|c|c|c|c|c|c|c|c|c|c|c|}
\hline \multicolumn{2}{|c|}{ Window size } & \multicolumn{5}{|c|}{ Average area } & \multicolumn{5}{|c|}{ Standard deviation } \\
\hline No & Length $\times$ normal & 1 & 2 & 3 & 4 & 5 & 1 & 2 & 3 & 4 & 5 \\
\hline 1 & $256 \times 128$ & 6.53 & 5.27 & 5.23 & 5.26 & 3.32 & 52.59 & 41.48 & 35.45 & 28.48 & 12.91 \\
\hline 2 & $256 \times 256$ & 5.90 & 4.61 & 4.96 & 4.89 & 3.31 & 53.05 & 41.57 & 38.20 & 32.15 & 14.01 \\
\hline 3 & $512 \times 64$ & 7.99 & 6.60 & 5.93 & 5.53 & 2.85 & 48.23 & 42.14 & 33.03 & 26.99 & 9.61 \\
\hline 4 & $512 \times 128$ & 6.75 & 5.85 & 5.40 & 5.11 & 3.35 & 53.91 & 45.10 & 35.38 & 28.03 & 13.62 \\
\hline 5 & $512 \times 256$ & 5.93 & 5.11 & 5.08 & 4.79 & 3.33 & 54.80 & 47.03 & 36.36 & 29.22 & 14.41 \\
\hline
\end{tabular}

grades of samples. After the window size is decided, the Eigen value with grade classification ability could be obtained. The most important task becomes selecting a suitable threshold to differentiate position. When the window size is $512 \times 128$ pixels, according to Table 1 with regard to average area the threshold can be decided as $6.00,5.50,5.30$ and 4.00 , respectively. Meanwhile, with regard to standard deviation the threshold can be decided as 48.00, 40.00, 30.00 and 20.00, respectively. That is to say, in the acquisition of fabric images, degree of seam smoothness can be deduced stably without taking into account the effect of sewing stitch position.

\section{The Size of Feature Window}

According to the ABEMD, two relative Eigen values in pixel were computed and normalised within an image. Thus, the Eigen value is constant with the changing of the window size. However, if the window size is too small, the image cannot include the information of sewing stitch, resulting in an inaccurate result. The difference between the image with sewing stitch or without sewing stitch influences the result, which leads to inaccurate calculation results. If the window size is too large, the larger data will influence the computer speed. Therefore, the proper size of feature window must be considered.

In order to study what window size for measuring sewing stitch is appropriate, we investigated the measurement result under different window sizes for five samples. The results of five kinds of window sizes are shown in Table 2. The window sizes are $128 \times 256,256 \times 256,64 \times 512,64 \times 512$ and $64 \times 512$, respectively. There are two kinds of direction in sewing, length direction as lengthwise sewing stitch and normal direction as crosswise sewing stitch. From Table 2, we found that there is no relationship between sewing stitch and average area when the size of length direction is less than 512 pixels, although there are positive correlations between sewing stitch wrinkle and standard deviation. Meanwhile, if the size of length direction is greater than 512 pixels, there are positive correlations between sewing stitch wrinkle and average area or standard deviation when the size of normal direction is 64,128 or 256 . As shown above, there is a range of window size suitable for analysing grades of sewing stitch. It is because when appropriate window size is used in the image, the proportion of sewing stitch part is differentiated from others.

As analysed above, the window size influences the results. Thus, selecting the window size according to the range suitable for all types of sewing stitches can be viewed as a general criterion.

\section{Conclusion}

In this paper, a novel classification method of assessing for garment sewing stitch based on ABEMD has been introduced. BIMF and residue of $A B E M D$ can be selected to represent sewing stitch appearances of garment, and the image processing can eliminate noise information associated with fibre, yarn and weave structures. Two parameters that characterise garment sewing stitches, AACS and SDCS, have been defined based on the grey value of pixels. It is demonstrated that the parameters are useful in revealing basic features of garment sewing stitches, and are effective in discriminating sewing stitch images in terms of the grades when used as inputs for the ABEMD. The performance of the algorithm on different garment status is significantly reliable. The approach is robust and self-adaptive. For future work, we will focus on optimising the algorithm for fabric image with different colours.

\section{Acknowledgments}

The authors were grateful for the financial support by the open project programme of Key Laboratory of Eco-Textiles (Jiangnan University), Ministry of Education, China (NO. KLET1113, NO.KLET1213), and the Fundamental Research Funds for the Central Universities of Jiangnan University (JUSRP211A51). 


\section{References}

[1] Amirbayat J. (1990). An energy approach to the instability problem of overfed seams, part 1, International Journal of Clothing Science \& Technology, 2(1), 21-25.

[2] Amirbayat J., Morton, M.L. (1990). An energy approach to the instability problem of overfed seams: part 2, International Journal of Clothing Science \& Technology, 2(2), 7-13.

[3] Zavec P.D., Gersak J., Demsar J., Bratko I. (2006). Predicting seam appearance quality, Textile Research Journal, 76, 235-242.

[4] Pasayev N., Korkmaz M., Baspinar D. (2012). Investigation of the techniques decreasing the seam slippage in chenille fabrics (Part I), Textile Research Journal, 82(9), 855-863.

[5] Pasayev N., Korkmaz M., Baspinar D. (2011). Investigation of the techniques decreasing the seam slippage in chenille fabrics (Part II), Textile Research Journal, 81(20), 20752081.

[6] Mousazadegan F., Saharkhiz S., Latifi M. (2012). Prediction of tension seam pucker formation by finite-element model, International Journal of Clothing Science and Technology, 24(2/3), 129-140.

[7] Stylios G., Parsons Moore R. (1993). Seam pucker prediction using neural computing, International Journal of Clothing Science \& Technology, 5, 24-27.

[8] Fan J., Leeuwner W. (1998). The performance of sewing threads with respect to seam appearance, Journal of Textile Institute, 89, 142-154.

[9] Dobilaite V., Juciene M. (2006). The influence of mechanical properties of sewing threads on seam pucker, International Journal of Clothing Science \& Technology, 18, 335-345.

[10] Inui S., Yamanaka T. (1998). Seam pucker simulation, International Journal of Clothing Science \& Technology, 10, 128-142.

[11] Inui S., Okabe H., Yamanaka T. (2000). Simulation of seam pucker on two strips of fabrics sewn together, International Journal of Clothing Science \& Technology, 13, 53-64.

[12] Hu J.L., Ma L., George B., Sai Keung Wong W., Zhang W. (2006). Modeling multi-layer seam puckering, Textile Research Journal, 76, 665-673.

[13] Samuel W.D., Poojitha V. (2010). A simple system for the online detection of skip/loop stitches in single needle lockstitch sewing machines, AUTEX Research Journal, 10(3), 69-72.

[14] Rengasamy R.S., (2011). Samuel Wesley D. Effect of thread structure on tession peaks during lock stitch sewing, AUTEX Research Journal, 11(1), 1-5.

[15] AATCC 88B-2011. (2012). Smoothness of seams in fabrics after repeated home laundering. AATCC Technical Manual of the American Association of Textile Chemists and Colorists, 114-117.

[16] Shiloha M. (1971). The Evaluation of seam-puckering, Journal of The Textile Institute, 62(3), 176-180.

[17] Kim H.A., Kim S.J. (2011). Seam pucker and formability of the worsted fabrics, Fibers and Polymers, 12(8), 10991105.

[18] Dobilaite V., Juciene M. (2010). Evaluation of seam pucker using shape parameters, Materials Science (Medziagotyra), 16(2), 154-158.
[19] Fan J., Liu F. (2000). Objective evaluation of garment seams using $3 D$ laser scanning technology, Textile Research Journal, 70(11), 1025-1030.

[20] Styliosa G., Sotomia J.O. (1993). Investigation of seam pucker in lightweight synthetic fabrics as an aesthetic property Part I: A cognitive model for the measurement of seam pucker, Journal of The Textile Institute, 84(4), 593-600.

[21] Styliosa G., Sotomia J.O. (1993). Investigation of seam pucker in lightweight synthetic fabrics as an aesthetic property Part II: Model implementation using computer 'Vision', Journal of The Textile Institute, 84(4), 601-610.

[22] Amirbayata J. (1992). Seams of different ply properties. Part I: Seam appearance. Journal of The Textile Institute, 83(2), 209-217.

[23] Mousazadegan F., Saharkhiz S., Latifi M. (2013). Seam pucker rating by deconvolution residual method, International Journal of Clothing Science and Technology, 25(3), 150-170.

[24] Yang X.B., Huang X.B. (2003). Evaluating fabric wrinkle degree with a photometric stereo method. Textile Research Journal, 73, 451-454.

[25] Yu W., Yao M., Xu B. (2009). 3-D surface reconstruction and evaluation of wrinkled fabrics by stereo imaging, Textile Research Journal, 79, 36-46.

[26] Zaouali R., Msahli S., El Abed B., Sakli F. (2007). Objective evaluation of multidirectional fabric wrinkling using image analysis. Journal of The Textile Institute, 98, 443-451.

[27] Sun J., Yao M., Xu B., Bel P. (2011). Fabric wrinkle characterization and classification using modified wavelet coefficients and support-vector-machine classifiers, Textile Research Journal, 81, 902-913.

[28] Liu J., Zhu B., Jiang H. (2013). Image analysis measurement of cottonseed coat fragments in $100 \%$ woven fabric, Fibers and Polymers, 14(7), 1208-1214.

[29] Jiang H., Liu J., Pan R., Gao W., Wang H. (2013). Autogeneration color image for fabric based on FFT, Industria Textila, 64(4), 195-203.

[30] Zhang X., Pan R., Liu J., Gao W., Xu W. (2011). Design gabor filters in the frequency domain for unsupervised fabric defect detection, Industria Textila, 62(4), 177-182.

[31] Dariush S., Mehdi H., Mohammad S., Zary R. (2012). Study of structural parameters of weft knitted fabrics on luster and gloss via image processing, Industria Textila, 63(1), 42-47.

[32] Huang N.E., Shen Z., Long S.R., Wu M.C., Shin H.H., Zheng Q., Yen N.C., Tung C., Liu H.H. (1998). The empirical mode decomposition and the Hilbert spectrum for nonlinear and non-stationary time series analysis, Proceeding R. Soc. Lond. A. 903-1005.

[33] Bernini M.B., Federico A., Kaufmann G.H., (2008). Noise reduction in degetal speckle pattern interferometry using bidimensional empirical mode decomposition, Applied Optics, 47(14), 2592-2598.

[34] Xu G.I., Wang X.T., Xu X.G. Improved bi-dimensional EMD and Hilbert spectrum for the analysis of textures, Pattern Recognition, 2009, 42, 718-734.

[35] Liu Z.X., Peng S.L. Boundary processing of bidimensional EMD using texture synthesis, IEEE Signal Processing Letters, 2005, 12, 33-36.

[36] Liang L.F., Ping Z.L. An edge detection algorithm of image based on empirical mode decomposition, in: Second International Symposium on Intelligent Information Technology Application, Shanghai, China, 2008, 1, 128-132. 
AUTEX Research Journal, Vol. 13, No 4, December 2013, DOI: 10.2478/v10304-012-0037-1 @ AUTEX

[37] Zheng Y., Zheng Q. Region-based image fusion method using bidimensional empirical mode decomposition, Journal of Electronic Imaging, 2009, 18(1):013008.
[38] Damerval C., Meignen S., Perrier V. A fast algorithm for bidimensional EMD, IEEE Signal Processing Letters, 2005,12(10), 701-704. 